\title{
Application of PSO algorithms for VPP operation optimization
}

\author{
D. Kaczorowska ${ }^{1}$, J. Rezmer ${ }^{1}$, T. Sikorski ${ }^{1}$, P. Janik ${ }^{2}$ \\ ${ }^{1}$ Faculty of Electrical Engineering \\ Wroclaw University of Science and Technology \\ Wybrzeze Wyspianskiego 27, 50-370 Wroclaw (Poland) \\ ${ }^{2}$ Tauron Ekoenergia \\ Obroncow Pokoju 2B, 58-500 Jelenia Gora (Poland) \\ e-mail: dominika.kaczorowska@pwr.edu.pl, jacek.rezmer@pwr.edu.pl, tomasz.sikorski@pwr.edu.pl, \\ przemyslaw.janik@tauron-ekoenegia.pl,
}

\begin{abstract}
The random character of generation of distributed energy resources and their increasing numbers cause problems with power balancing in distribution grids. One approach to mitigate the balancing issue is deployment of a virtual power plant VPP with accordingly dimensioned components, i.e. renewable generation and storages. It is also necessary to control the power flow in a way that production and consumption balance would not be threaten. From an investor's perspective, profits and costs are important. The components of a VPP installation, such as generators and storage systems must meet the conditions for optimal economic and technical performance. Due to the increasing number of optimization variables, and thus the complexity of the objective function, it is necessary to use advanced tools for effective solution for optimization problems. The paper presents the optimization of PV and electric energy storage for selected operational scenario using particle swarm algorithms. The proposed method allows for substantial improvement of power losses in the power grid and decrease of necessary investment. Predefined power consumption target profiles were reached even with reduced PV power and storage capacity. Real production and consumption curves were used in the presented analysis.
\end{abstract}

\section{Key words}

Energy storage, particle swarm optimization, photovoltaic system, virtual power plant.

\section{Introduction}

Similar to a microgrid [1] - [3], a low voltage virtual power plant (VPP) bundles generators and storage units, where VPP and electric power consumers interact actively to meet a predefined production or achieve robust balance between generation and consumption in an autonomous power system. All actors are equipped with communication and control units, e.g. power electronic converters governing the flow of energy. Such structure can operate as an autonomous standalone system, but it also can be connected to the public distribution grid. One of the main advantages of proposed VPP is the ability to store excessive electric energy to follow a predefined generation curve. An optimized energy storage enables increased utilization of energy produced by volatile renewable energy sources [4] - [5].

The renewable energy targets adopted in recent years, e.g. 20-20-20 EU goals, have contributed not only to the increase of clean and sustainable power generation, but also to the development of control methods, including those based on artificial intelligence algorithms [6] - [7]. According to the adopted control method, microgrids are classified as centrally controlled or as endowed with distributed control. In the case of distributed control scheme autonomous operation of VPP including renewable generation and storage is desired. To achieve this goal, local control units should be equipped with artificial intelligent algorithms [10].

The paper presents the concept of control strategy for a small low voltage VPP, which was optimized using PSO (particle swarm optimization) method. A well-designed and optimized control algorithm, i.e. operational scenario, guaranties efficient and effective utilization of all VPP components (PV and storage) serving a predefined production curve or load curve. Smart meters carry out power flow measurements that are transmitted to the control unit. Then, the control scenario uses these measurements along with predefined operational objectives.

In the nodes of a VPP installation, power flow is constantly measured and communicated to the control unit. The operational scenario addresses objective and constraining functions, e.g. power losses minimization, reduction of distribution grid use, voltage control in nodes, etc.

With sufficient generation and storage capacities, an optimized VPP operation can execute economical strategies underlying energy exchange with the distribution network [11]. The optimization procedure for 
a VPP is a multi-criterion task with multiple possible versions. An example of such a multi-criterion approach is the minimization of electricity delivery costs along with maximization of renewable energy share and a protective use of storage unit, i.e. reducing the number of charging cycles.

A predefined operational scenario is crucial at the conceptual stage of a VPP design. Numerous parameters should be settled, e.g. power rating of renewable generators, charging/discharging characteristics of storages, cross-sections of cables, fault protection settings, etc. It also indirectly influences the parameters of other components [9].

The second important aspect of an operational scenario is the direct control the energy flow to and from the storage unit based on real-time measurements. Such a prospective scenario is then linked to short-term prediction methods for energy production and consumption estimation [12]. If needed, the parameters of the operational scenario can be then modified by particle swarm optimization.

This paper presents research results including theoretical considerations and practical simulations aimed at an operational scenario for carefully sized components of a VPP. The control scenario for energy flow in the VPP works with real data. The power generation values were measured in a photovoltaic research installation rated 15 $\mathrm{kW}$, and the predefined production target (load) is a real consumption of an office building on the campus of the Wroclaw University of Science and Technology.

\section{VPP serving a load in a microgrid}

One of the crucial challenges imposed by increased share of distributed renewable generation is undoubtedly the power flow management between major components in a distribution grid [11]. An important issue is the selection of optimal VPP parameters regarding generators and energy storages allowing for a predefined operational scenario.

The operational scenario proposed in this paper defines the energy flow to and from the VPP comprising PV generator, storage unit and load. The installation is connected to the public distribution grid.

A general assumption in the proposed operational scheme is full utilization of energy generated by the photovoltaic source. Thus, in the first place, the photovoltaic generator covers the demand of the load (predefined production goal). The energy storage unit acts as a buffer, collecting the excess energy generated by the PV and returning it when the power demand is greater than the momentary production of the photovoltaic generator. The connection of the installation to the power system feeds the load when the sum of the power generated in PV and available from the storage unit is too small to balance the flow. Secondly, it gives the possibility of more flexible control of the energy flow. Despite apparent simplicity of the system, the control scenario is not at all obvious, and choosing the right set of parameters makes it possible to optimize the operation of the system, minimizing the losses, increasing power quality, adapting sizing of the photovoltaic generator and the storage unit.

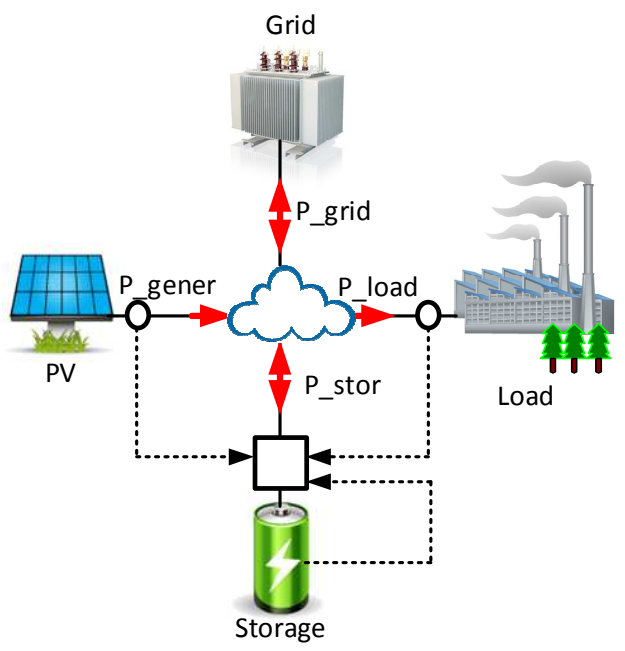

Fig. 1. Power flow control scheme.

An important functional block of the proposed VPP is the storage unit. The type and technology of storage unit imply important parameters, such as maximum storage capacity, e.g. maximum charging and discharging power, charging and discharging characteristics [13]. Fig. 2 shows an example of charging and discharging characteristics of a chemical type storage. Important features of these two characteristics are the maximum and minimum power, the maximum and minimum energy and the coefficient responsible of the slope shape in these curves.

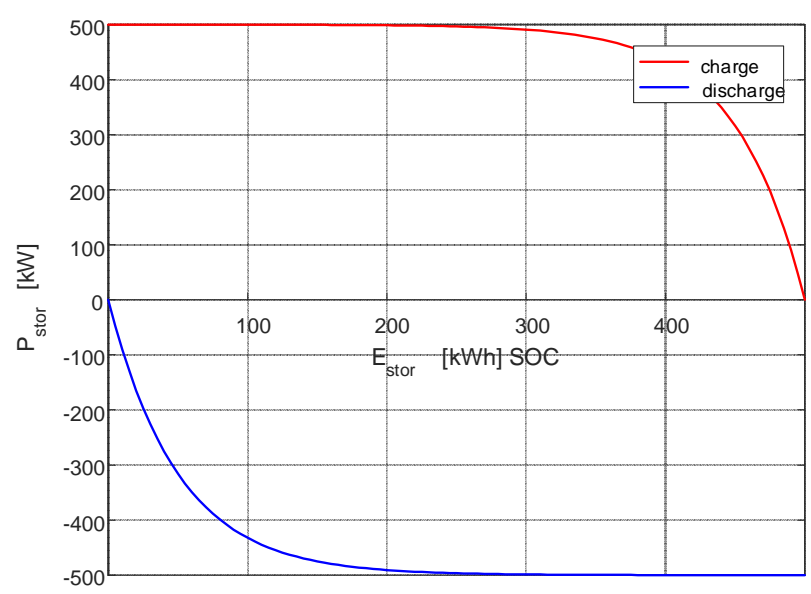

Fig. 2. Charging and discharging characteristic of the storage unit.

Voltage levels, i.e. voltage stability at particular installation nodes, as well as energy losses resulting from current flows between components, are very important aspects of the analysis of a particular control scenario of power flow. Such analysis results, in both DC and AC approaches, can be used for optimization of VPP components and for setting of parameters of the power flow scenario.

The control rules and physical constrains for power flow in the studied VPP are compactly described through equations (1) to (7). 


$$
\begin{gathered}
I_{\text {load }}^{2}=\frac{P_{\text {load }}}{R_{\text {load }}} ; \quad U_{p p}=I_{\text {load }} R_{\text {load }}+U_{\text {load }} \\
-I_{\text {store }}^{2} R_{\text {stor }}+I_{\text {stor }} U_{p p}-P_{\text {stor }}=0 \\
U_{\text {stor }}=U_{p p}-I_{\text {stor }} R_{\text {stor }} \\
-I_{\text {gener }}^{2} R_{\text {gener }}+I_{\text {gener }} U_{p p}-P_{\text {gener }}=0 \\
U_{\text {gener }}=U_{p p}-I_{\text {gener }} R_{\text {gener }} \\
I_{\text {grid }}=-I_{\text {load }}-I_{\text {stor }}-I_{\text {gener }} \\
U_{\text {grid }}=U_{p p}-I_{\text {grid }} R_{\text {grid }} ; \quad P_{\text {grid }}=I_{\text {grid }} U_{\text {grid }}
\end{gathered}
$$

where: $P_{\text {grid }}$ - power of the public grid, $P_{\text {gener }}$ - power of the generation, $P_{\text {load }}$ - power of the load, $P_{\text {stor }}$ - power of the storage, $U_{\text {grid }}$ - voltage in the grid node, $U_{\text {gener }}$ - voltage in the generation node, $U_{\text {load }}$ - voltage in the load node, $U_{\text {stor }}$ voltage in the storage node, $U_{p p}$ - voltage in the common coupling point, $I_{\text {grid }}$ - current in the grid line, $I_{\text {gener }}$ current in the generator line, $I_{\text {load }}$ - current in the load line, $I_{\text {stor }}$ - current in the storage line, $R_{\text {grid }}$ - the grid line resistance, $R_{\text {gener }}$ - the generator line resistance, $R_{\text {load }}$ - the load line resistance, $R_{\text {stor }}$ - the storage line resistance

For calculation of power flows, especially in the case of analyses of flows in the AC network, a special calculation package MATPOWER working in the Matlab environment was used.

\section{VPP Operational Scenario}

The proposed scenario for controlling the energy flow from VPP is particularly focused on the storage unit. The correct choice of a control variant and according control options is based on the energy flow measurements and settles the charging or discharging ratio of the storage unit. The control is performed using a power electronic converter installed at the storage. Schematic representation of a power flow control scenario is shown in Fig. 3.

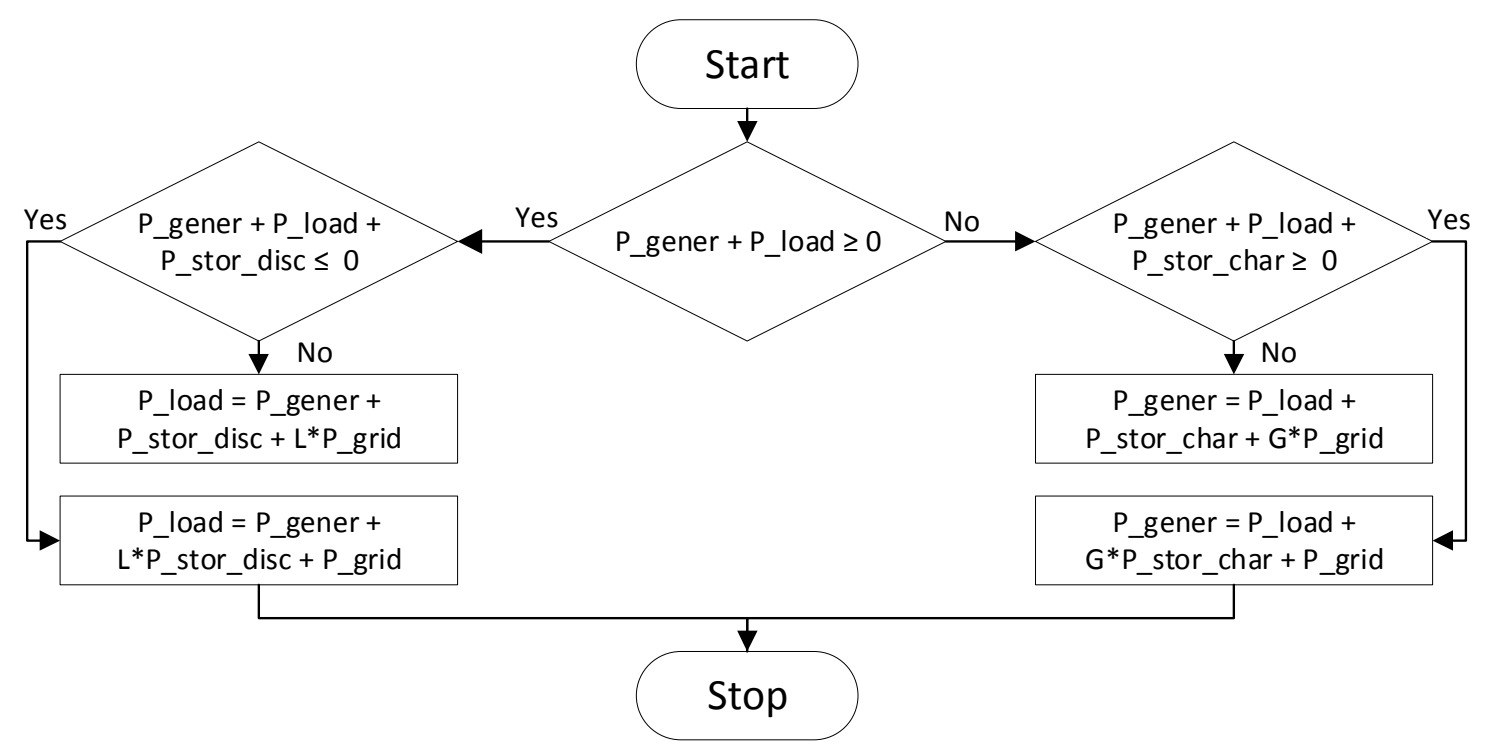

Fig. 3. Schematic representation of a power flow control scenario in a microgrid.

\section{Optimization}

Computational intelligence, i.e. particle swarm intelligence, was used for parameters optimization of the VPP components and operational scenario. The basis for the operation of the swarm is the exchange of information, experience and knowledge, which enables the cooperation of all individuals in the swarm. Ultimately, the position of the swarm leader in the multidimensional search space indicates an optimal solution [8]. The general scheme of the algorithm are described in (8) and (9).

$$
\begin{gathered}
V_{i}(k+1)=D V_{i}(k)+C_{1} R_{1 i}\left[P_{i}(k)-X_{i}(k)\right]+ \\
+C_{2} R_{2 i}\left[S(k)-X_{i}(k)\right] \\
X_{i}(k+1)=X_{i}(k)+V_{i}(k+1)
\end{gathered}
$$

where: $V_{i}$ - velocity of i-th particle, $X_{i}-$ location, $C_{1}, C_{2}-$ acceleration coefficients, $P_{i}$ - best location of i-th particle, $S$ - best location of the swarm leader. $R_{1}, R_{2}-$ random numbers from the range $\langle 0,1\rangle, D$ - inertia coefficient.

The objective (adaptation) function evaluates the subsequent solutions obtained in subsequent iteration steps. Its minimum value is the criterion for choosing the best solution. During the conducted analyzes, a multicriteria optimization was applied. Objective function is based on minimizing the energy exchange with the distribution network, minimizing the installed PV power and minimizing the capacity of the storage with different weighting factors.(10).

$$
\varepsilon=w_{1} \sum_{k=0}^{N} P(k)_{g r i d+}+w_{2} E_{\text {stor } \max }+w_{3} P_{P V}
$$


where: $P(k)_{\text {grid }}$ - power from the public grid in a given period of time, $E_{\text {stor max }}$ - maximum storage capacity of the storage unit, $P_{P V}-$ installed PV power, $w_{1}, W_{2}, W_{3}-$ weighting coefficients.

\section{Results}

Based on the proposed scenario for controlling the energy flow to and from the storage unit, the system components were optimized using particle swarm algorithms.

In some cases, due to the limited number of charging cycles defining the lifetime of storage, the energy exchange must be limited. Analyzed case refers to the situation in which the parameters of the PV system are set and the VPP interaction with the distribution network is defined as well. It is done by assigning constant values to the scenario parameters controlling energy flow. The parameters of the storage unit and PV installation were computed using the proposed optimization. By selecting the $L$ and $G$ scenario coefficients, it is possible to dimension the PV and storage parameters so that the amount of power transferred to and from the storage unit is minimal. Dimensioning was done for the extreme values of coefficients in the scenario and for the average values as well.

The resulting $P_{\text {gener }}$ power generation curve and $P_{\text {load }}$ load power curve are shown in Fig. 4. This figure also includes the power curve of the storage unit $P_{\text {stor }}$ and the power exchanged with the distribution grid $P_{\text {grid. }}$ Fig. 5 shows the variations in the load power curve and the power fed from the public distribution grid into the VPP infrastructure. A significant reduction in energy exchange with the distributor network can be observed, in comparison to the operation of the load without local generation and storage of energy. Fig. 6 shows the energy cumulated in storage.

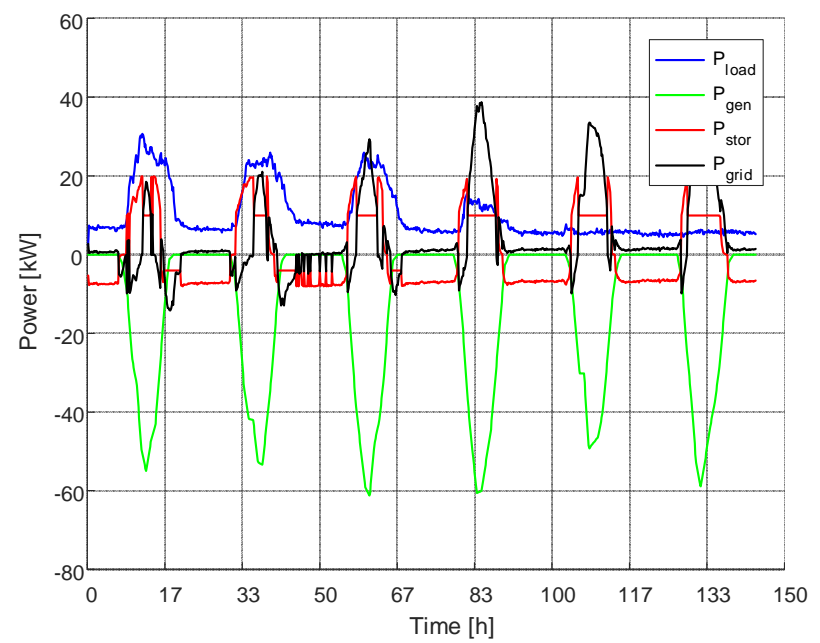

Fig. 4. Characteristics of load power, PV generation, storage power and distribution grid power.

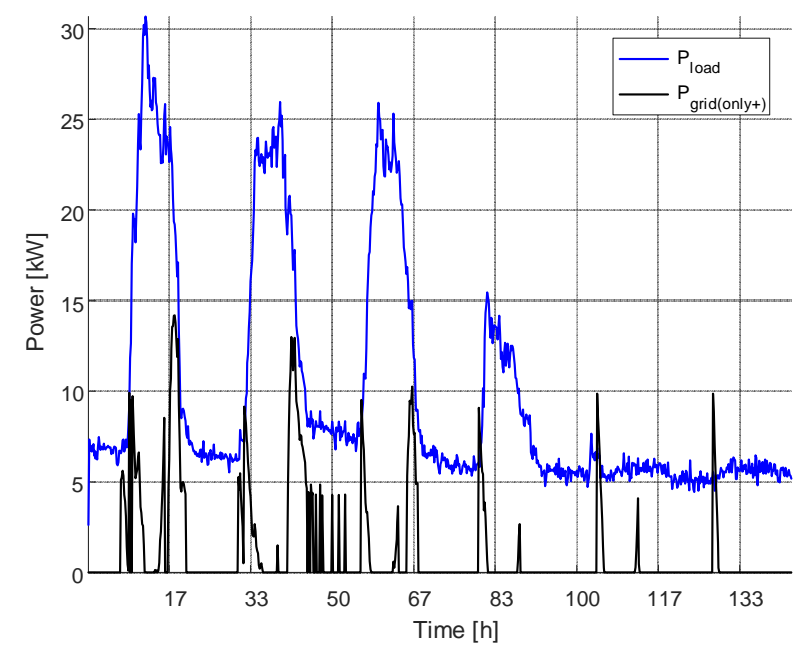

Fig. 5. Load power characteristic and power input from the grid power.

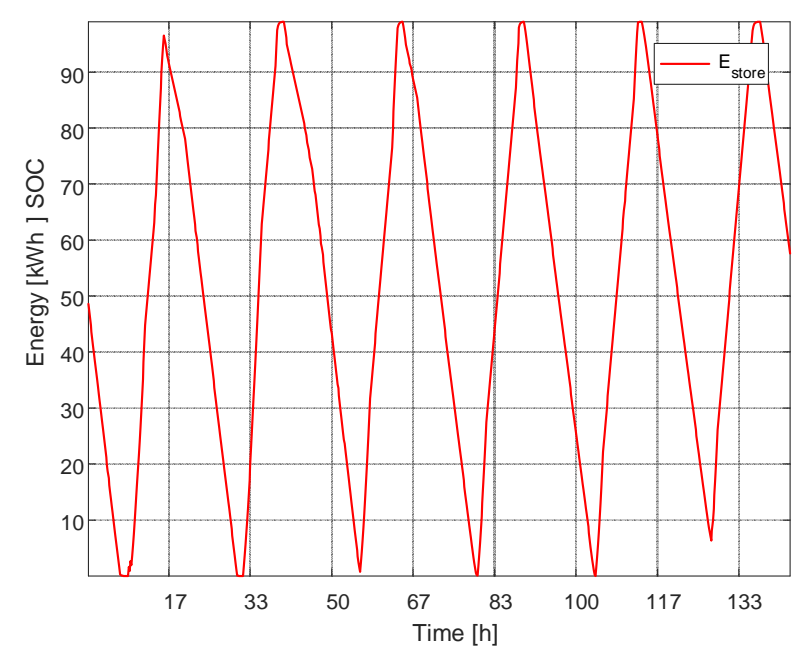

Fig. 6. The energy curve of the storage unit.

The results of the selection of storage size and PV system dimension are presented in Table I.

Table I. - Storage size and PV system dimension in accordance to $\mathrm{L}$ and $\mathrm{G}$ coefficients

\begin{tabular}{|l|c|c|c|c|}
\hline Coefficients values & N_PV & E_max & P_char & P_disch \\
\hline $\mathrm{L}=\mathrm{G}=0.0$ & 4.04 & 77.78 & 20.00 & 26.41 \\
\hline $\mathrm{L}=\mathrm{G}=0.5$ & 5.00 & 97.25 & 19.91 & 8.09 \\
\hline $\mathrm{L}=\mathrm{G}=1.0$ & 5.00 & 102.11 & 13.73 & 6.72 \\
\hline
\end{tabular}

The parameters that are taken into account during optimization are not only the magnitude of the generation, the load variation, the power of exchange with the distribution network and the parameters and characteristics of the reservoir, but also the parameters of the scenario itself controlling the flow of energy in the microgrid. The analysis must also take into account changes in the nodes of the microgrid and the energy losses in feeders and lines [14]. These parameters may also be taken into account when constructing the objective function for an optimization algorithm. For example, Fig. 9 shows changes in the voltage in the nodes of the installation. 


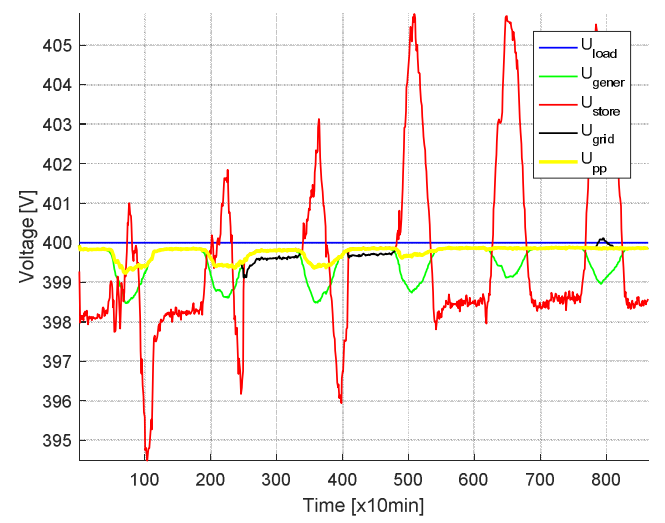

Fig. 9. Voltage curves at nodes in the microgrid.

\section{Conclusion}

Approaches to system modelling based on computational intelligence (PSO, AG, neural networks) are nowadays more and more commonly used, in addition to standard optimization algorithms used previously. This is due to the fact, that multi- criterial objective function handled with standard minimum seeking methods fail, being stuck in an unsatisfactory local minimum. Algorithms based on mathematical intelligence are resistant to these problems, more robustly seek the global minimum of the objective function. In addition, their continuous development allows for more accurate and robust solutions delivered timely. The algorithms used showed fast convergence to the minimum of the objective function in the first iterative steps, followed by a significant slowdown in further iterations. In the future, a combination of different optimization methods can be considered to shorten the calculation time, along with further fine-tuning of the algorithms used. A shorter calculation time would allow further research with an increased number of optimization variables. Further to include automatic scenario selection as an optimization parameter.

Even with a simple scenario, focused on the power flow control to and from the storage unit, artificial intelligence methods are very useful in sizing the system components guaranteeing minimal power exchange with external power system. Dimensioning of microgrid components using artificial intelligence is a difficult issue, especially choosing the right objective function and corresponding constrains. Including additionally investment costs into this procedure could bring significantly different results [12]. However, the argument for using proposed methods, i.e. PSO is the ability of multi-criterial evaluation of the objective function and low sensitivity to the local minima, which often occur in such optimization problems.

\section{Acknowledgement}

This work was done under project: "Study of cooperation between the distribution grid, photovoltaic generator system and energy storage" supported by the Wroclaw University of Science and Technology under a support scheme for young researchers.

This work was done under the project "Developing a platform for aggregating generation and regulatory potential of dispersed renewable energy sources, power retention devices and selected categories of controllable load" supported by European Union Operational Programme Smart Growth 2014-2020, Priority Axis I: Supporting R\&D carried out by enterprises, Measure 1.2: Sectoral R\&D Programmes, POIR.01.02.00-00-0221/16, performed by TAURON Ekoenergia Ltd. under Polish Sectoral Program PBSE coordinated by The National Center of Research and Development in Poland.

\section{References}

[1] A. Rojas, T. Rousan, "Microgrid Control Strategy: Derived from Stakeholder Requirements Analysis" IEEE Power and Energy Magazine, 2017, vol. 15, issue 4 pp. 72 - 79.

[2] D. Ton, J. Reilly, "Microgrid Controller Initiatives: An Overview of R\&D by the U.S. Department of Energy" IEEE Power and Energy Magazine, 2017, vol. 15, issue 4, pp $24-31$.

[3] G. Joos; J. Reilly; W. Bower; R. Neal, "The Need for Standardization: The Benefits to the Core Functions of the Microgrid Control System", IEEE Power and Energy Magazine, 2017, vol. 15 , issue 4, pp. $32-40$.

[4] G.K. Venayagamoorthy, R.K. Sharma, P.K. Gautam, A. Ahmadi, "Dynamic Energy Management System for a Smart Microgrid", IEEE Transactions on Neural Networks and Learning Systems, 2016, vol. 27, issue 8, pp. $1643-1656$.

[5] D. U. Sauer, J. Kowal, T. Blank, "Energy storage technologies for grids with high penetration of renewables energies and for grid connected PV systems," in 23rd European Photovoltaic Solar Energy Conference and Exhibition, pp. 26742687.

[6] Peng Li, Duo Xu, Zeyuan Zhou, Wei-Jen Lee, Bo Zhao, "Stochastic Optimal Operation of Microgrid Based on Chaotic Binary Particle Swarm Optimization" IEEE Transactions on Smart Grid Year, 2016, vol. 7, issue 1 pp. 66 - 73.

[7] R. Rigo-Mariani, B. Sareni; X. Roboam, "Integrated Optimal Design of a Smart Microgrid With Storage", IEEE Transactions on Smart Grid, 2017, vol. 8, issue 4 pp. 1762 - 1770.

[8] D. Kaczorowska, J. Rezmer, Particle swarm algorithm for microgrid optimization. W: 2018 Innovative Materials and Technologies in Electrical Engineering (i-MITEL). Danvers, MA : IEEE, cop. 2018. s. 1-4.

[9] A. Gubanski, D. Kaczorowska, Power flow optimization between microgrid and distribution system. W: 2018 Innovative Materials and Technologies in Electrical Engineering (iMITEL). Danvers, MA : IEEE, cop. 2018. s. 1-4.

[10] P. Piagi, R. H. Lasseter, "Autonomous control of microgrids," in Power Engineering Society General Meeting, pp. $8-15$.

[11] C Li, X. Zhu, G. Cao, S. Sui, M. Hu "Dynamic modeling and sizing optimization of stand-alone photovoltaic power systems using hybrid energy storage technology," Renewable Energy, vol. 34, pp. 815-826, June 2009. Available: http://www.sciencedirect.com/science/article/pii /S0960148108001523

[12] S. R. Vosen, J. O. Keller, "Hybrid energy storage systems for stand-alone electric power systems: optimization of system performance and cost through control strategies," International Journal of Hydrogen Energy, vol. 24, pp. 1139-1156, December 1999. Available: http://www.sciencedirect.com/science/article /pii/S036031999800175X.

[13] B. J. Szymanski, K. Kompa, L. Roslaniec, A. Dmowski, J. Szymanski, "Operation of photovoltaic power systems with energy storage," in Compatibility and Power Electronics (CPE), 2011 7th International Conference-Workshop, pp. 86-91.

[14] J. von Appen, T. Stetz, M. Braun, " Local Voltage Control Strategies for PV Storage Systems in Distribution Grids," IEEE Transactions on Smart Grid, vol. 5, pp. 1002-1009, February 2014. Available: http://ieeexplore.ieee.org/abstract/document/ 
6733334/ 